\title{
Steadfast Energy Proficient Sensor Node Activation System in Wireless Networks Lifetime Enhancement
}

\author{
R. Saravanakumar ${ }^{*}$, Dr. N. Mohankumar² \\ ${ }^{1}$ ECE/OAS Institute of Technology and Management, Pulivalam, Tiruchirappalli, Tamilnadu, India \\ ${ }^{2}$ ECE/SKP Engineering College, Thiruvannamalai, Tamilnadu, India \\ Email: "rsksusi@gmail.com
}

Received 12 March 2016; accepted 25 April 2016; published 28 April 2016

Copyright (C) 2016 by authors and Scientific Research Publishing Inc.

This work is licensed under the Creative Commons Attribution International License (CC BY). http://creativecommons.org/licenses/by/4.0/

(c) $\underset{\mathrm{EY}}{\mathrm{i}}$ Open Access

\begin{abstract}
The wireless sensor network (WSN) is one of the budding exploring areas and fast rising fields in wireless communications. The sensor nodes in the network are generally small-size, low-cost, lowpower and multi-function capabilities. Wireless sensor networks (WSNs) are used for various applications; since numerous sensor nodes are usually deployed on remote and inaccessible places, the employment and preservation should be easy and scalable. Sensor nodes in the field being run out of energy quickly has been an issue and many energy efficient routing protocols have been proposed to solve this problem and preserve the long life of the network. This paper work proposes a hierarchical based node activation routing technique which shows energy efficiency. This technique selects cluster head with highest residual energy in each communication round of transmission to the base station from the cluster heads. Hierarchical based node activation routing technique with different levels of hierarchy simulation results prolongs the lifetime of the network compared to other clustering schemes and communication rounds of simulation increase significantly.
\end{abstract}

\section{Keywords}

Wireless Sensor Networks, Hierarchical Routing, Energy Efficiency, Clustering, Node Activation Scheme, Network Lifespan

\section{Introduction}

Since swift innovation in electronics industry, small low-cost battery-powered wireless sensors have already

"Corresponding author.

How to cite this paper: Saravanakumar, R. and Mohankumar, Dr.N. (2016) Steadfast Energy proficient Sensor Node Activation System in Wireless Networks Lifetime Enhancement. Circuits and Systems, 7, 402-416.

http://dx.doi.org/10.4236/cs.2016.74035 
started to make a brunt on the communication with the physical globe. WSNs can be deployed in a wide geological space to monitor physical observable fact with acceptable accuracy and reliability. The sensor nodes can observe various parameters, which can be effectively used in commercial, military, and environmental applications. WSNs consist of copious battery-powered devices and energy-efficient network protocols must be designed. Owing to large network size, limited power supply, and unreachable remote employment surroundings, the WSNs based protocols are dissimilar from the conventional wireless protocols [1] [2]. The central part operation of WSNs is to collect process data from the individual sensor nodes, and transmit the essential data to the base station for further analysis and processing. At present there are several energy efficient communication models and protocols that are designed for specific applications. The routing algorithm proposed is suitable for continuous monitoring of numerous prevalent sensor nodes, which are outsized space from the base station. There have been reflective studies on WSNs [3] at some points in the recent years. Since the sensors in the network have limited battery power, ostentatious lifetime of a network is the basic aim of designing an energy efficient routing protocol. Clustering concept routing protocols aim to achieve energy efficiency. The whole network is divided into clusters with a cluster head node for each cluster. The data from sensors inside a cluster are aggregated at cluster head. This eliminates a lot of redundancy in packet forwarding. Low Energy Adaptive Clustering Hierarchy (LEACH) [4] is considered to be yardstick protocol in Clustering/Hierarchical based protocols.

The structure of the remaining paper is as follows: Section 2 briefly describes the related work. In Section 3, base of hierarchical cluster-based routing protocol with problem statement and contribution is described. Section 4 states the proposed routing protocol details. Section 5 indicates the proposed routings validation of simulation results from hierarchical routing techniques. Finally, Section 6 concludes the paper and provides directions for some future work.

\section{Related Work}

Various methods for minimizing energy consumption in wireless sensor network have been proposed such as by Heinemann et al. [5] who described the LEACH protocol as a hierarchical self organized cluster based approach for monitoring application. The data collection area of the data is randomly divided into clusters. LEACH protocol normally utilize the time division multiple access (TDMA) system to transmit data from the sensor nodes to the cluster head. Then cluster head aggregates the data and transmits it to the base station for processing. One of the features of LEACH is localized coordination and control for the formation and operation of clusters. The cluster head rotate randomly. In [6] Lindsey et al. came about the proposition of PEGASIS which is a conservatory of LEACH. PEGASIS protocol in WSN eliminates the overhead of energetic cluster formation created by LEACH. In this protocol, the nodes transmit to the cluster head and transmission of data is done by the cluster head, which is selected in a rotational manner, to the base station. When compared to LEACH protocol PEGASIS protocol is establish to save more energy and is more vigorous in node failure.

Muruganathan et al. [7] developed a protocol that creates clusters of the similar size and uses multi-hop routing between cluster head and the base station. The cluster head forward the last hop is selected randomly from the sets of cluster heads to diminish the consignment of cluster head which are located nearest to the base station. In [8], Wei Li proposed a geometric programming model to extend the network lifetime of the sensor network by clustering sensor nodes into groups. He developed an iterative method for solving the geometric programming by choosing the optimal location of cluster heads. The optimum mentioned in his proposition refers to minimizing energy consumption based on inter-sensor network under specific constrained. Clustering of approaches is useful in the monitoring of habitat and environs. This however, necessitates the use of continuous stream of sensor data. Ye, Heinemann and Estrin [9] gave a description of a contention based medium access protocol, which minimizes energy consumption in wireless sensor network by using implicit clusters.

Wei Cheng et al. [10] proposed a narrative adaptive energy efficient clustering algorithm for wireless sensor network. Al-Karaki and Kamal [11] made an assessment of the routing technique in wireless sensor network and mentioned that hierarchical routing technique has the several advantages related to efficient communication.

\section{Problem Statement and Contribution}

More researches done on the features of hierarchical technique have been found to offer better approach to effectively prolong the life time of WSNs. In WSNs, the available energy is mainly consumed by data transmission, signal processing and hardware operation of the system. It is proved that $70 \%$ of energy consumption is 
caused during data transmission process [12]. Therefore, the process of data transmission should be optimized in order to maximize the network lifetime. The data transmission in sensor network can be optimized by an efficient ways of data aggregation and using efficient routing protocol. To improve the wireless sensor network lifespan, an efficient data aggregation protocols which help to eradicate redundant data transmission in a wireless sensor network must be in place. Usually, a multi-hop approach is necessary to facilitate data collection by one node which can then forward the received data to a neighbor node that is nearer to the base station. The receptive node aggregates the data collected and also forwards it on. But the process of aggregation and forwarding of data directly from a source to the base station causes significant energy wastage as each node in the entire network is actively involved in the operation. There is a situation of clustering where each node sends data to the cluster head and then the cluster head performs aggregation on the received raw data and then sends it to the BS [13]. This approach also consumes a substantial amount of energy which needs to be improved upon. To have minimum level of energy consumption in WSNs, this paper proposed an approach whose aim of cluster head selection is based on the highest predicted residual energy after the following round and the shortest distance via the closest neighboring cluster head to the base station. Since hierarchical routing technique offers a better scalability and efficient communication approach and its concept can also be effectively used to design energy efficient routing protocol in WSNs. This paper proposes the new energy efficient routing protocol with node activation method to improve the network lifetime of wireless sensor network by applying node activation method in a hierarchical routing protocol and by implementing a new way of cluster head selection and rotation. The validation of the proposed idea, simulation and results are analyzed.

\section{Overview of Routing Techniques}

A variety of protocols were proposed for prolonging the life of WSNs and for routing the correct data to the base station. But each protocol has disadvantages and is not suitable for area monitoring applications. These protocols cannot be used directly due to resource constraints of sensor nodes because it has limited battery power, communication capability and computational speed. New protocols are being researched and fashioned to eliminate the problem faced in WSNs. The various protocols can be classified as location-based, data-centric or hierarchical.

The location based routing technique for WSNs depends on location information of sensor nodes for estimation of distance between two specific nodes to deduce energy consumption. The protocol for information via negotiation (SPIN) [14] data are named using metal-data that highly describes the characteristics of the data which is the key feature of SPIN. In flooding routing protocol each sensor node receives data and then sends them to the neighbors by broadcasting, unless a maximum number of hops for the packet are reached or the destination of packet is achieved. The advantage of SPIN is that the topological changes are localized since each of the sensor nodes needs to know only its single-hop neighbors. Besides SPIN's data announcement method cannot guarantee the delivery of data.

Hierarchical routing in WSN involves the arrangement of clusters in form of hierarchy when sending information from the sensor nodes to the base station. Energy consumption is gained using hierarchical routing by implementing multi-hop communication [15] for a specific cluster and thus performing aggregation of data and fusion in a way that decreases the number of data carried across the network to the sink. Based on the residual energy in the sensor nodes the cluster formation and election of a cluster head process is being done. A very good example of a hierarchical routing protocol is based on low-energy adaptive clustering hierarchy (LEACH) routing protocol. The LEACH with node scheduling concept is explained in [16].

The Table 1 shows the comparison of the different routing protocols in terms of scalability, lifetime, data diffusion and power required in WSN. From Table 1, it is understand that hierarchical technique offers an approach to energy minimization and scalability features in WSNs [17]-[19].

\section{The Proposed Hierarchical Routing without/with Node Activation Schemes}

Main points adopted in the proposed hierarchical routing protocol are

- It is based on the principle of clustering algorithm.

- Modified LEACH protocol in terms of hierarchical data transfer.

- Clusters are formed by geographical area division.

- Cluster head selection is based on the residual energy comparison in each round. 
Table 1. Comparison of routing techniques.

\begin{tabular}{ccccc}
\hline \multirow{2}{*}{$\begin{array}{c}\text { Comparison of routing } \\
\text { techniques }\end{array}$} & Parameter & Data centric & Location & Hierarchical \\
\cline { 2 - 5 } & Scalability & Limited & No & Good \\
& Lifetime & Long & Long & Long \\
& Data diffusion & No & No & Yes \\
& Power required & Limited & Limited & High \\
\hline
\end{tabular}

- Geographical formation of cluster sizes is based on equal segmentation of area as in Figures 4-6.

- Adopted the node scheduling or activation algorithm.

\subsection{Algorithm Description of the Proposed Routing Protocol}

Proposed hierarchical routing has been implemented as shown in Figure 1 for without node activation, with node activation scheme 1 (sleep/active) and with node activation scheme 2 (sleep then active). Each scheme has three levels as mentioned below.

Level 1: One cluster with one head (Overall area)

- Level 1 has overall area of $300 \mathrm{~m} \times 300 \mathrm{~m}$ with 250 sensor nodes, which is considered as one single cluster and under the principle of clustering concept one cluster head can be selected in each round of data communication by comparing the residual energy of individual sensor node as shown Figure 3.

Level 2: Two clusters with two heads (Overall area is divided in to two groups)

- In level 2, the overall area space is divided into two segments and 250 sensor nodes are randomly deployed in two groups with two heads are elected as per the residual energy basis in each round as shown in Figure 4. Level 3: Three clusters with three heads (Overall area is divided in to three groups)

- In level 3, the overall area space is divided in to three segments and the 250 sensor nodes are randomly deployed in three groups with three heads are elected as per the residual energy basis in each round as shown in Figure 5.

Data communication from each sensor node to cluster head and cluster head to base station is under multi hop communication by finding shortest path.

In hierarchical routing without node activation scheme, all the three levels, sensor nodes are only in active mode. In hierarchical routing with node activation scheme 1 (Active/Sleep), all the levels, inside the cluster half numbers of the sensor nodes are activated to active mode and remaining sensor nodes are activated to sleep mode. After the first round of data communication, active nodes are activated to sleep mode and sleep nodes are activated to active mode and vice versa the change of mode is occur in each round. In hierarchical routing with node activation scheme 2 (Active then Sleep), all the levels, inside the cluster, half of the sensor nodes from the available sensor nodes are activated to active mode and the remaining half are activated to sleep mode. In simulation all the active nodes are drain out of its energy after some rounds then the sleep nodes are become automatically activated to active nodes.

\subsection{Implementation of Hierarchical Routing for without/with Node Activation Methods}

The proposed hierarchical routing protocol is basically based on the principle of clustering algorithm. The data transmission at the network layer being the core area of interest which is the modified LEACH protocol in terms of hierarchical data transfer with the employment of energy prediction technique for selection of cluster head via any shortest path to the base station. In this method clusters are formed geographical area segmentation. Geographical formation of cluster sizes is based on equal segmentation of node deployment area.

Apart from the one cluster formation which makes use of the entire sensors area, other formation such as two and three clusters formation involves equal segmentation of area. The two and three cluster formation is known as second level and third level hierarchy respectively. The sensor nodes are randomly deployed in the field as shown in Figure 2. The hierarchical routing sensor node topology for level 1 to 3 is shown in Figures 3-5. 


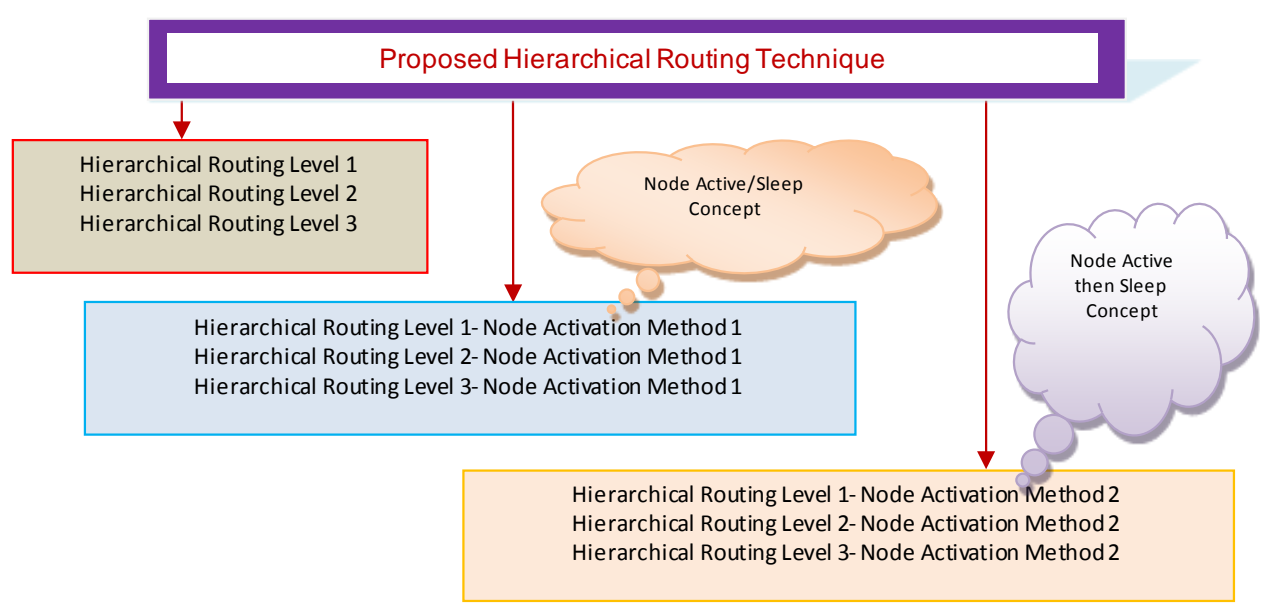

Figure 1. Proposed hierarchical routing.

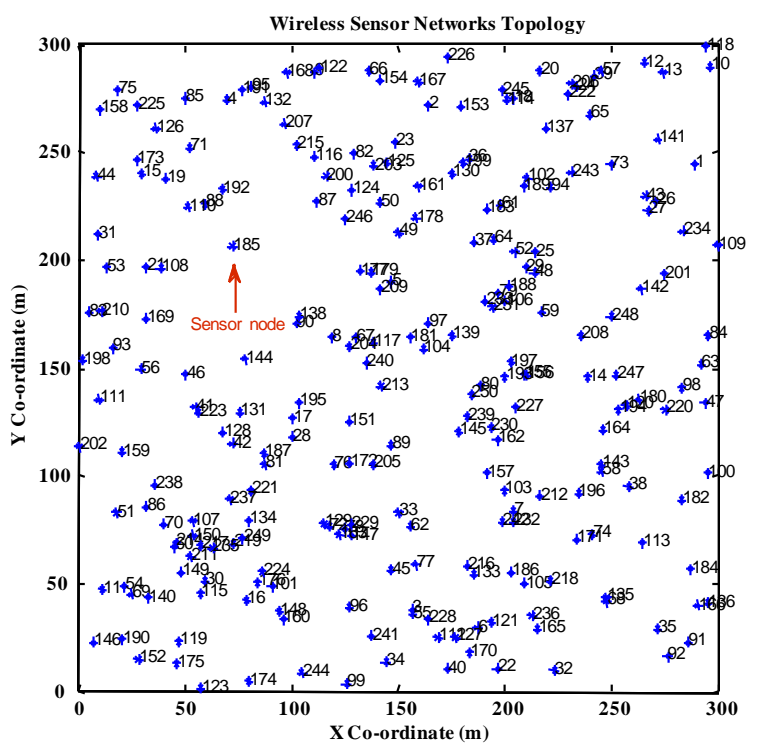

Figure 2. Basic wireless sensor network topology.

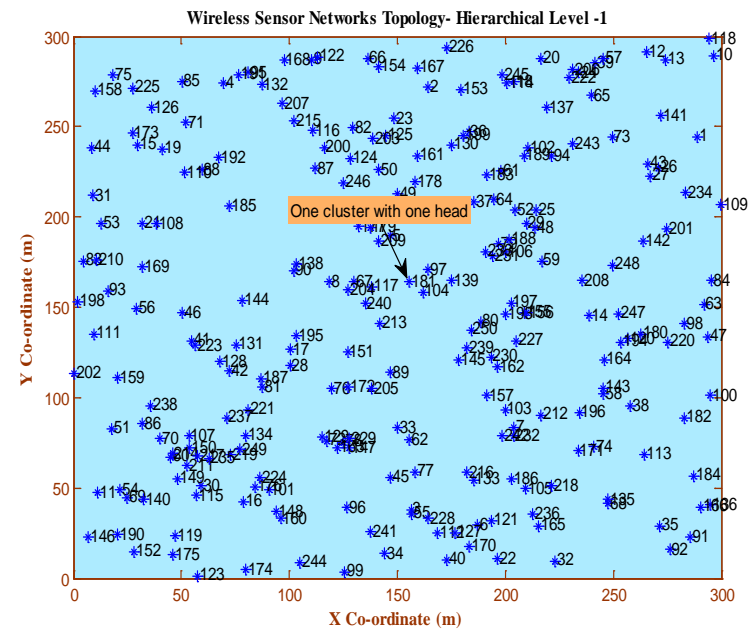

Figure 3. Hierarchical routing level 1. 


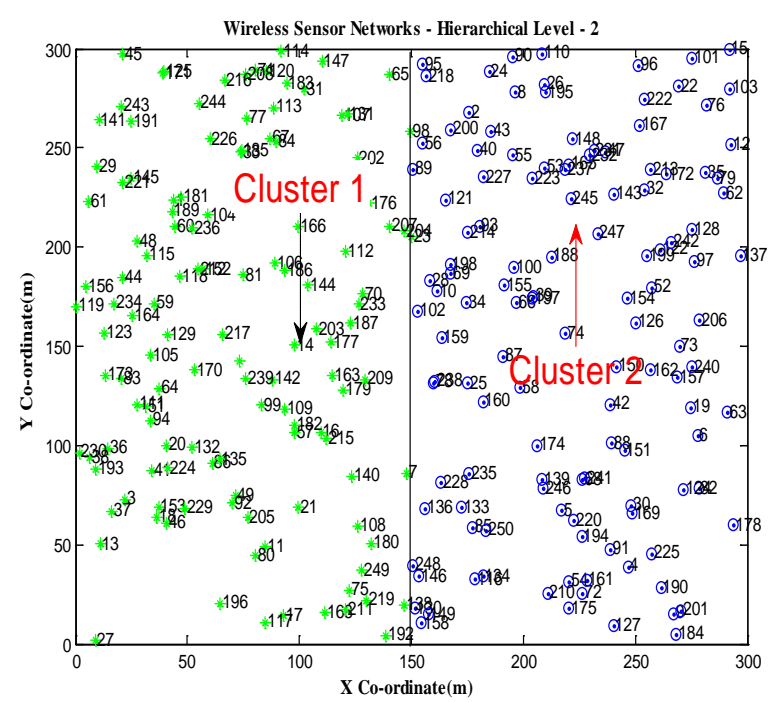

Figure 4. Hierarchical routing level 2.

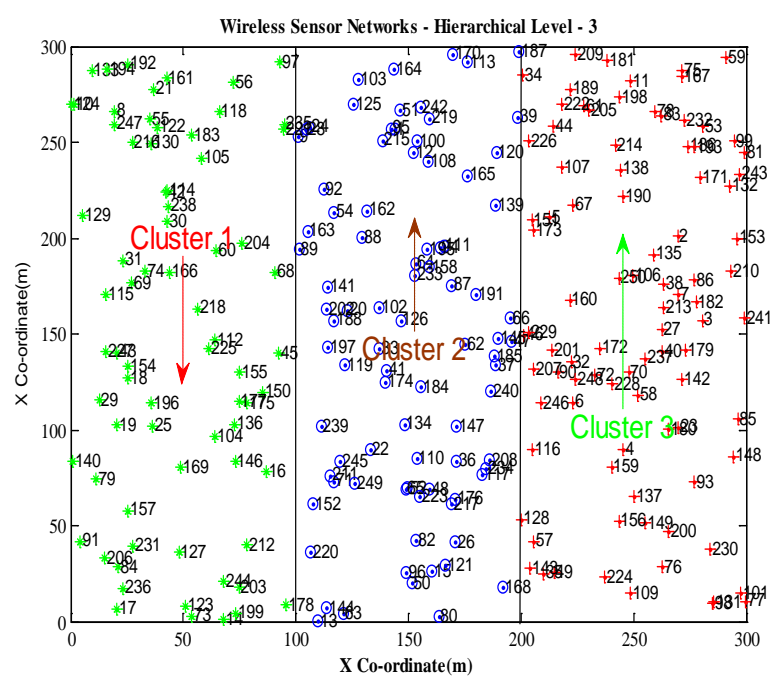

Figure 5. Hierarchical routing level 3.

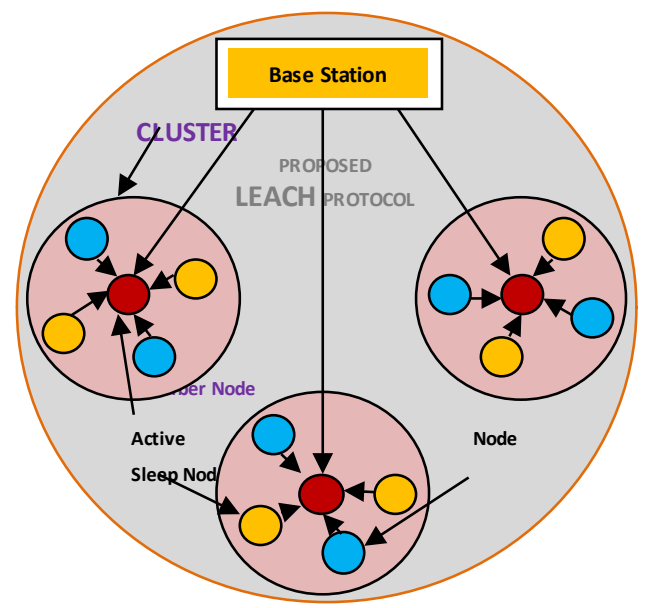

Figure 6. Node scheduling or activation scheme in hierarchical routing. 


\section{Hierarchical Routing with Node Scheduling Concept}

The basic concept of hierarchical routing is being implemented along with node activation method which is exhibited in Figure 6. Along with hierarchical routing two methods of node activation is incorporated to get minimum consumed energy and prolonging the lifetime of entire wireless sensor networks. The idea behind the method 1 is node active/sleep concept and method 2 is node active and sleep concept.

Node Activation Method 1:

In all the levels the procedure of forming cluster and cluster head are followed by LEACH protocol. In method 1 , in all levels, available sensor nodes are being activated under the active/sleep concept, which is nothing but in each cluster half number of nodes are being active and the remaining sensor nodes are being sleep mode. Under this condition the simulation obtained results shows that the lifetime is absolutely increased. Each round the active sensor nodes are changes its mode to sleep and sleep to active. Up to the energy level comes to zero this procedure being done.

Node Activation Method 2:

Method 2 has similar operation of LEACH protocol and in all levels available sensor nodes are being activated under the active and sleep concept, which is nothing but in each cluster half number of nodes are being active and the remaining sensor nodes are being kept in sleep mode. The entire available active sensor nodes are drain out of energy and then the sleep modes in each cluster comes to active and the same procedure is end with the total energy drain out of the entire node in the network.

\section{Validation with Justification of Simulation Results}

Hierarchical routing is implemented in its level one, two and three and the consolidated graph represents the difference, out of these levels the third level is giving improved lifetime than the other two levels. The proof is shown in Figure 7. In each level of hierarchical routing the available residual energy diagram after 400 round is shown in Figures 8-10. The residual energy of the individual node available in the network after 400 round is clearly depicted and in Figure 11, the third level hierarchical routing representation is obviously giving more remaining energy which is higher than the other two levels.

In method 1 node activation scheme of active/sleep concept is introduced in hierarchical routing level 1, 2 and 3. The network lifetime and its residual energy representation diagrams depicted in from Figures 11-16. From the residual energy diagram after 400 rounds by the implementation of node active/sleep concept, the level three hierarchical routing with node activation concept give the best result of energy savings to improve the lifetime of the network.

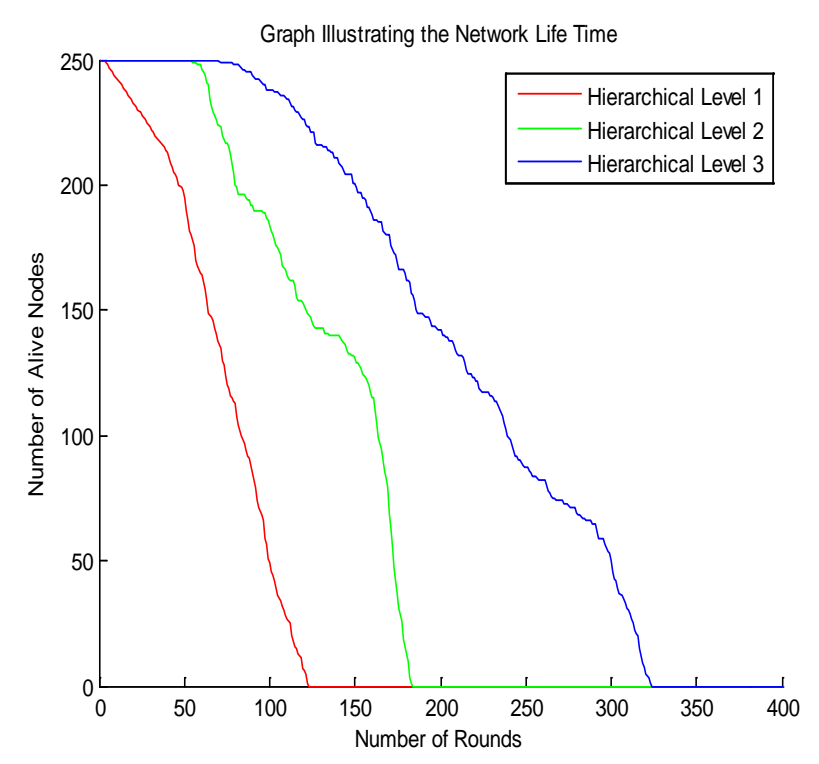

Figure 7. Hierarchical routing level 1, 2 and 3 network lifetime comparison. 


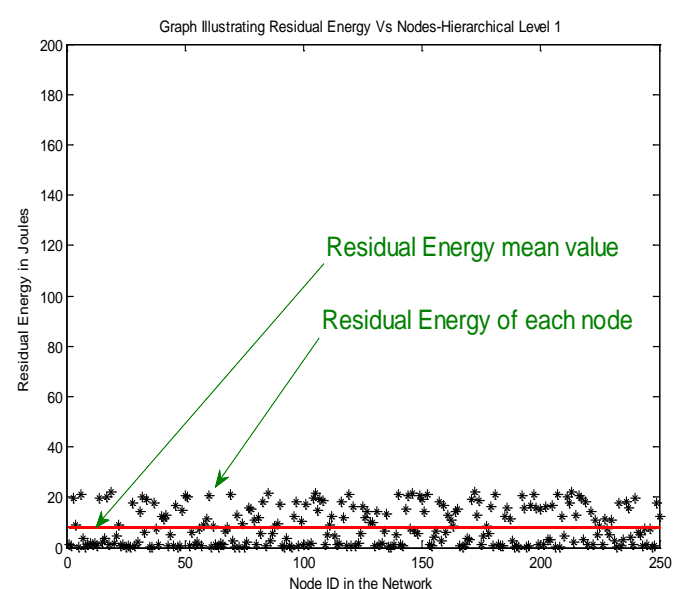

Figure 8. Residual energy representation for hierarchical routing level 1.

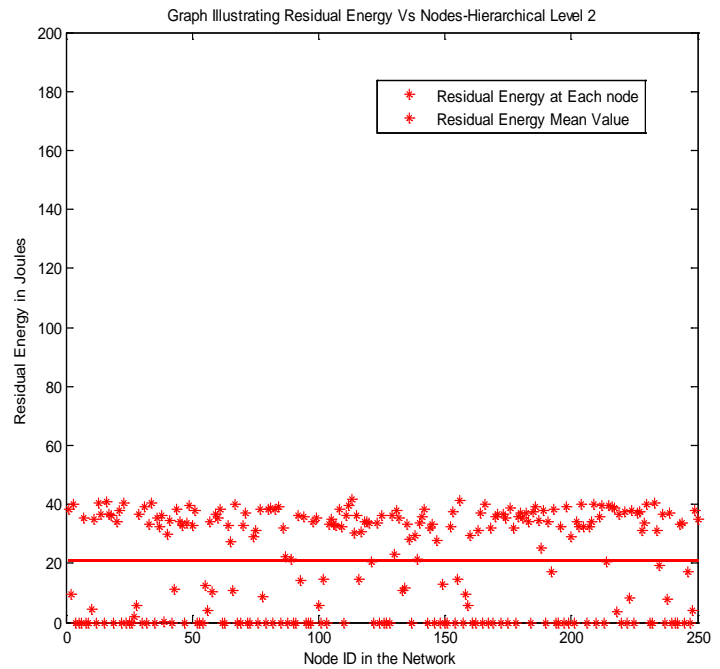

Figure 9. Residual energy representation for hierarchical routing level 2.

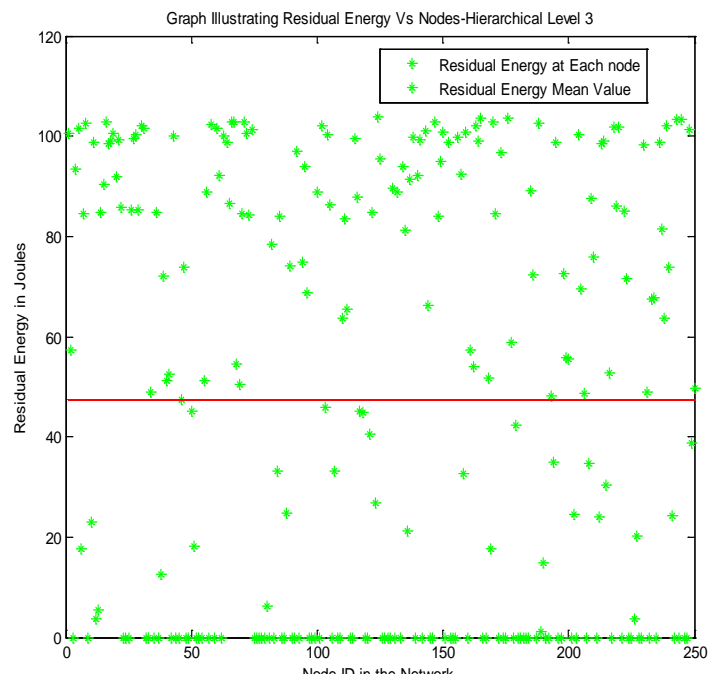

Figure 10. Residual energy representation for hierarchical routing level 3. 


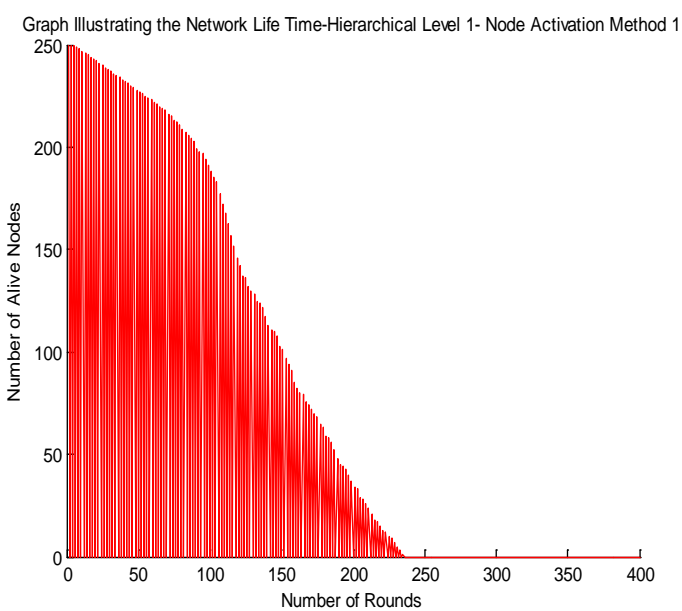

Figure 11. Network lifetime of hierarchical routing level 1 with node activation method 1 .

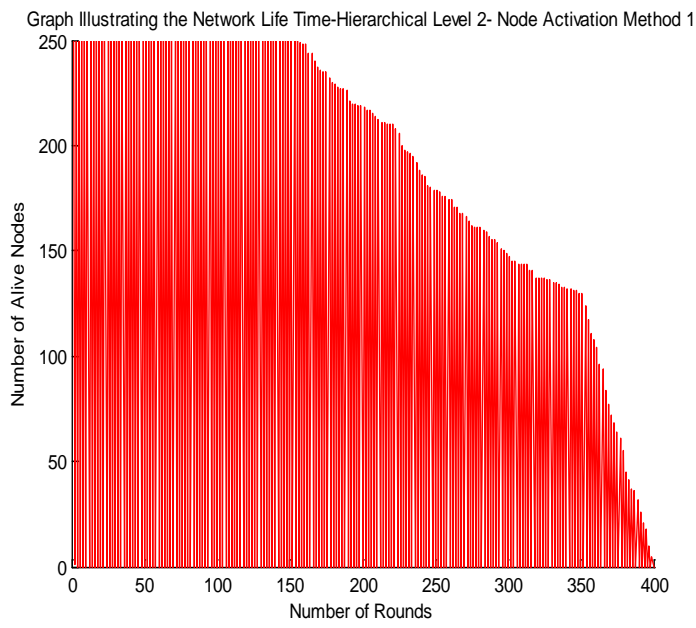

Figure 12. Network lifetime of hierarchical routing level 2 with node activation method 1 .

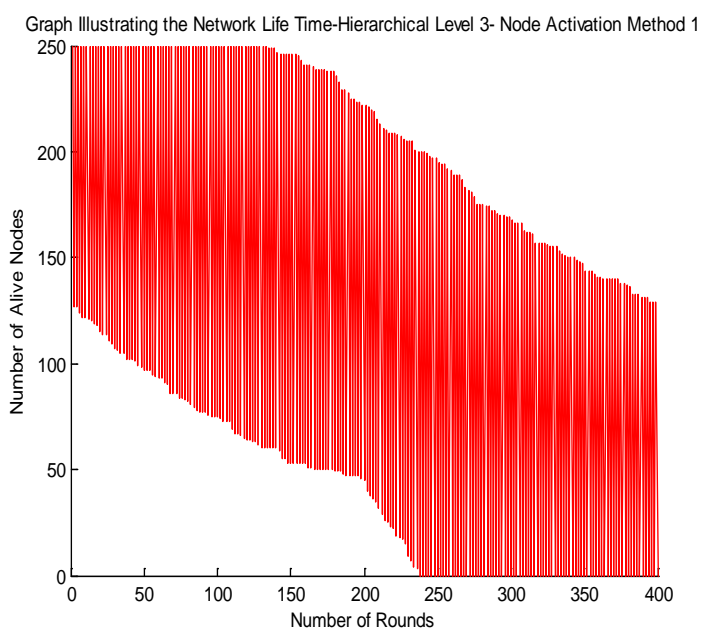

Figure 13. Network lifetime of hierarchical routing level 3 with node activation method 1 . 


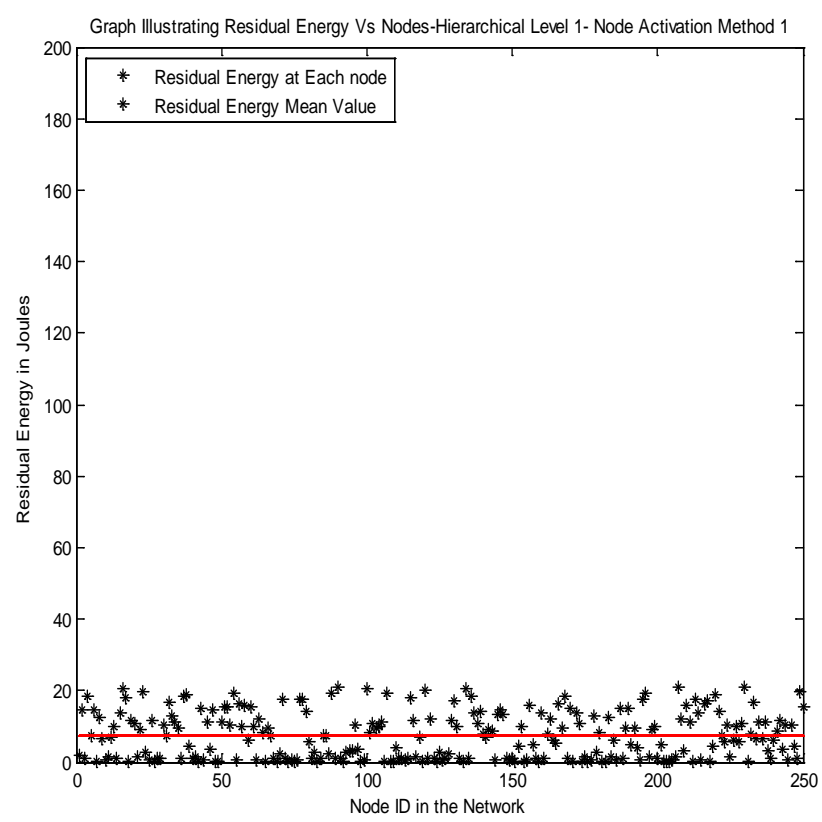

Figure 14. Residual energy of hierarchical routing level 1 with node activation method 1 .

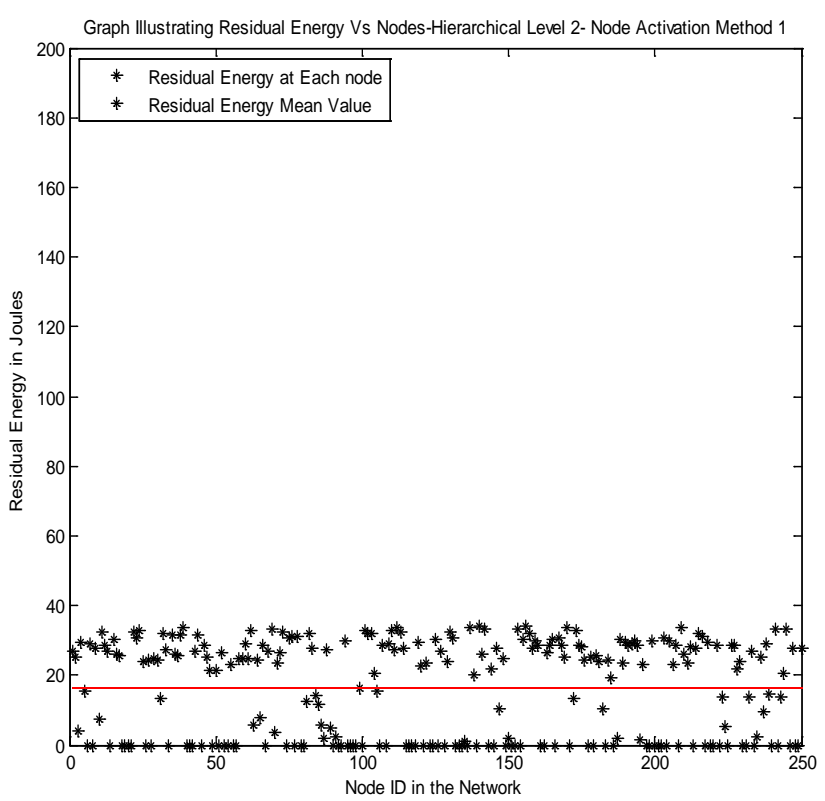

Figure 15. Residual energy of hierarchical routing level 2 with node activation method 1 .

In Figure 7 of hierarchical routing levels 1, 2 and 3 and its LEACH protocol based operations, under simulation work the level 3 three clusters with three cluster head concept is giving best performance of live nodes against with number of rounds. Level 1 ends with 125 rounds of all dead nodes, level 2 ends with 180 rounds of all dead nodes lost and level 3 ends with 325 rounds of dead nodes. So, the level 3 hierarchical routing yield the best lifetime improvement against the number of rounds. The residual energy representation for three levels are depicted in Figures 8-10 and hierarchical routing level 3 energy representation have more remaining energy compare to other two levels.

From Figures 11-13, the hierarchical routing with node activation methods 1 (active/sleep) level 1, 2 and 3 
implementation is shown and the simulation work for level 3 is giving best performance of live nodes against with number of rounds than the other two levels. The residual energy representation for three levels are depicted in Figures 14-16 and hierarchical routing with node activation methods 1 (active/sleep) level 3 energy representation have more remaining energy compare to other two levels.

From Figures 17-19, the hierarchical routing with node activation methods 2 (active then sleep) level 1, 2 and 3 implementation is shown and the simulation work for level 3 is giving best performance of live nodes against with number of rounds than the other two levels. The residual energy representation for three levels are depicted in Figures 20-22 and hierarchical routing with node activation methods 2 (active then sleep) level 3 energy representation have more remaining energy compare to other two levels.

The residual energy of all the methods is tabulated as standard mean values in Joules in Table 2. By observation

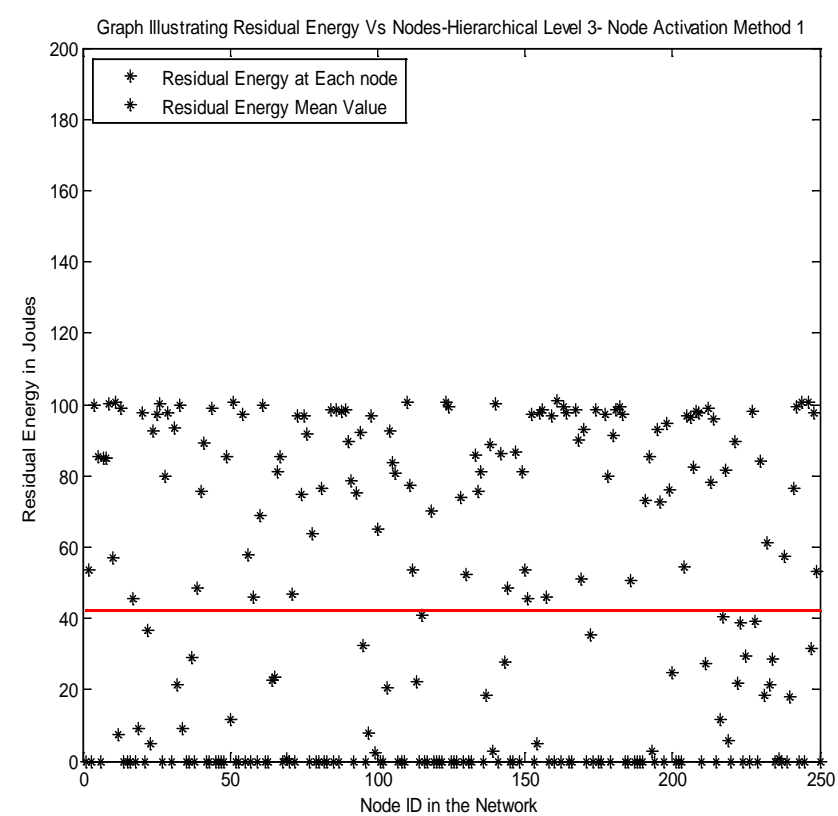

Figure 16. Residual energy of hierarchical routing level 3 with node activation method 1 .

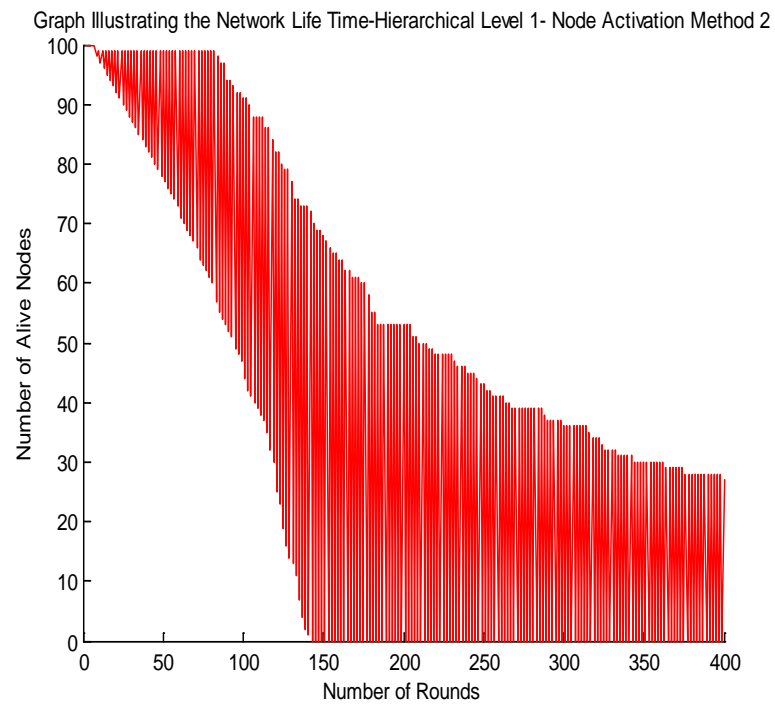

Figure 17. Network lifetime of hierarchical routing level 1 with node activation method 2. 


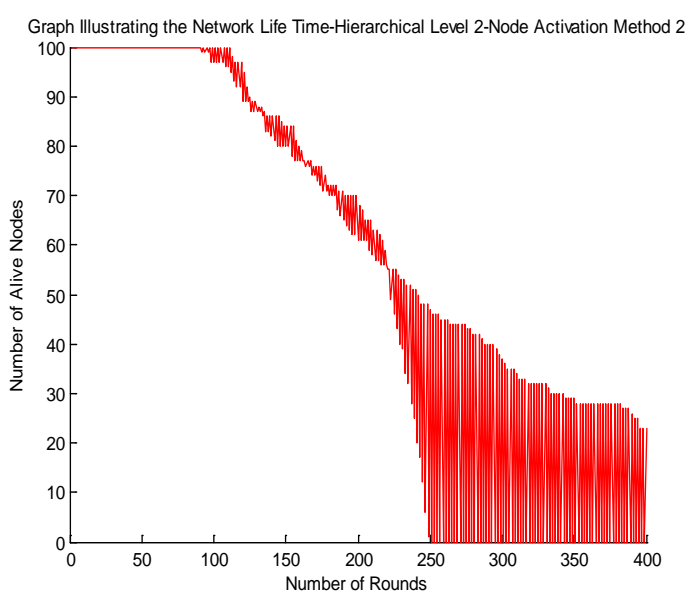

Figure 18. Network lifetime of hierarchical routing level 2 with node activation method 2 .

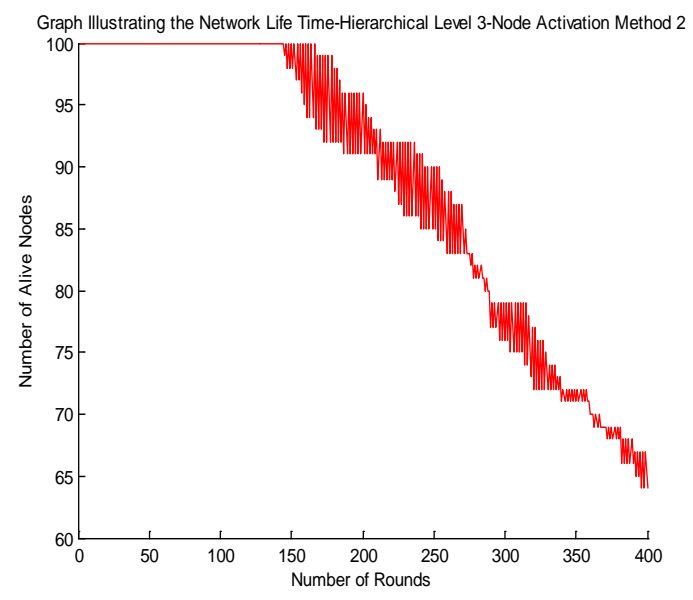

Figure 19. Network lifetime of hierarchical routing level 3 with node activation method 2 .

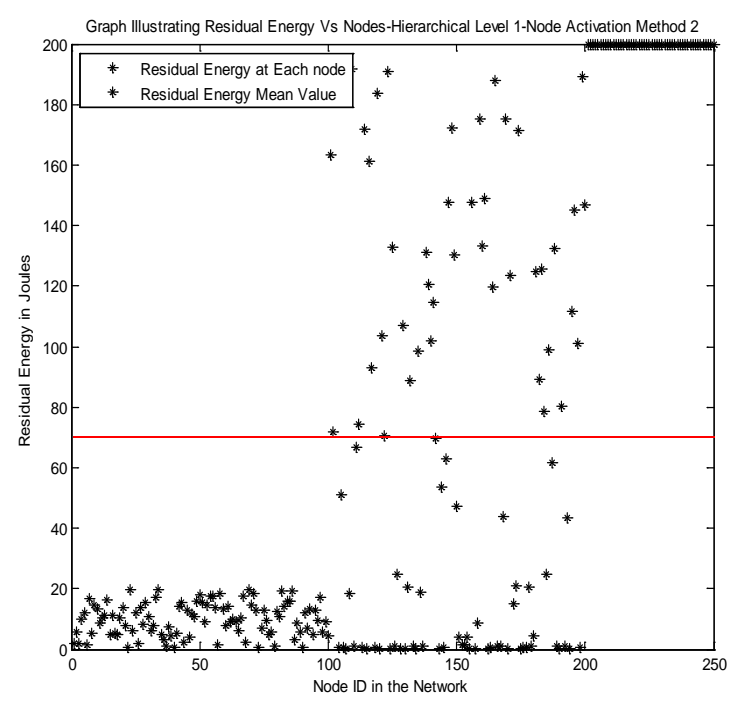

Figure 20. Residual energy of hierarchical routing level 1 with node activation method 2 . 


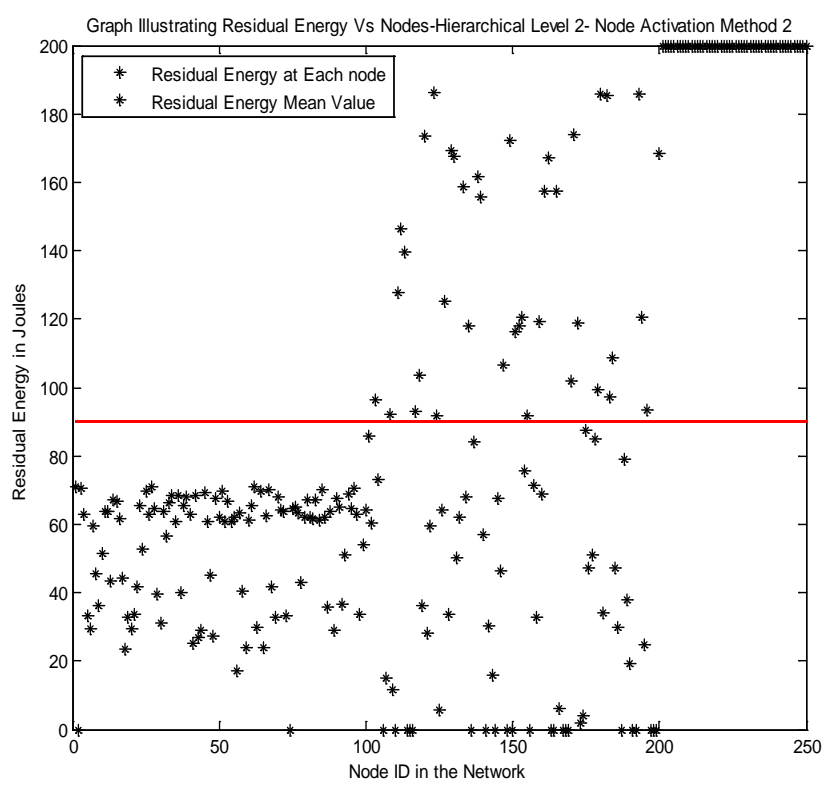

Figure 21. Network lifetime of hierarchical routing level 3 with node activation method 2 .

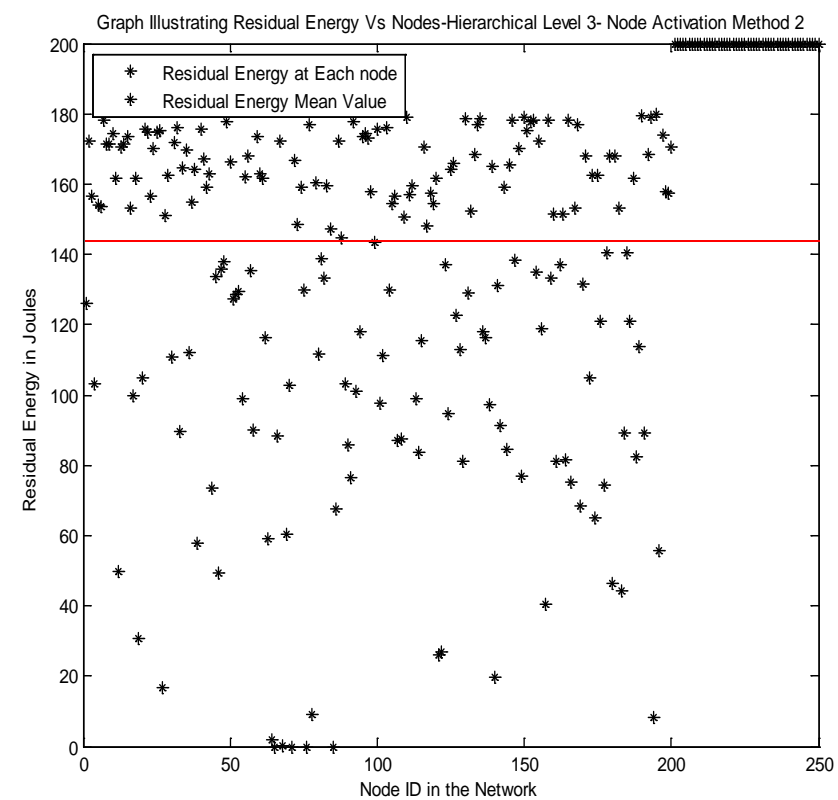

Figure 22. Residual energy of hierarchical routing level 1 with node activation method 2 .

the hierarchical routing level 3 node activation method 2 (active then sleep) standard mean value is giving the minimum energy consumped and having high value of mean. It is understood that the higher value is representing the remaining energy available in each sensor node after 400 round of simulation process. Table 3 indicates the details of simulation parameters used in simulation.

\section{Conclusion}

New concept through hierarchical routing technique based on the development of work in node deployment geographical area has been divided as segments and within the segment; the deployed sensor nodes and its cluster 
Table 2. The residual energy of standard mean value of all methods

\begin{tabular}{|c|c|c|}
\hline \multirow{2}{*}{$\begin{array}{c}\text { The standard mean value of } \\
\text { all methods }\end{array}$} & \multicolumn{2}{|l|}{ Various techniques } \\
\hline & Name of the method & Standard mean value $(\mathrm{J})$ \\
\hline & Hierarchical Routing Level 1 & 07.8692 \\
\hline & Hierarchical Routing Level 2 & 12.0860 \\
\hline & Hierarchical Routing Level 3 & 41.9168 \\
\hline & Hierarchical Routing Level 1- Node Activation Method 1 & 07.9992 \\
\hline & Hierarchical Routing Level 2- Node Activation Method 1 & 16.3419 \\
\hline & Hierarchical Routing Level 3- Node Activation Method 1 & 42.3501 \\
\hline & Hierarchical Routing Level 1- Node Activation Method 2 & 70.0472 \\
\hline & Hierarchical Routing Level 2- Node Activation Method 2 & 90.1922 \\
\hline & Hierarchical Routing Level 3- Node Activation Method 2 & 143.8999 \\
\hline
\end{tabular}

Table 3. Simulation parameters

\begin{tabular}{lcc}
\hline $\begin{array}{c}\text { The simulation } \\
\text { parameters }\end{array}$ & Various techniques & Quantity \\
\cline { 2 - 3 } & Name of the parameter & 250 \\
Total number of nodes in field & 200 \\
Initial energy of each node $\mathrm{E}_{\text {in }}$ in Joules & 100 \\
Packet size $\mathrm{k}$ in bytes & 50 \\
Amplifier coefficient $\mathrm{E}_{\text {amp }}$ in pico Joule per bit & 100 \\
Base station position in meters & $(150,150)$ \\
Topology area of the network in meters & $300 \times 300$ \\
\hline
\end{tabular}

formation with cluster head selection are implemented. In the implemented proposed routing protocol and its simulation results for hierarchical routing technique level 1, level 2 and level 3 for without and with node activation scheme, the level three hierarchical routing technique with node scheduling or node activation scheme exhibits its best results of energy consumption and increased lifetime of network in terms of number of rounds and dead nodes than other two levels. Inside the hierarchical routing technique and its levels, the node activation method 1 (active/sleep) and 2 (active and sleep) are incorporated in normal hierarchical routing levels 1, 2 and 3 in such a way to improve the lifetime of the network. The entire simulation results are obtained and out of that, hierarchical routing level 3 with node activation method 2 results is proved as extremely the best for the improved network lifetime and residual energy in terms of the number of live sensor nodes and dead nodes.

\section{References}

[1] Estrin, D., Govindan, R., Heidemann, J. and Kumar, S. (1999) Next Century Challenges: Scalable Coordination in Sensor Networks. Proceedings of the International Conference on Mobile Computing and Networks (MobiCom), 263-270. http://dx.doi.org/10.1145/313451.313556

[2] Estrin, D., Culler, D., Pister, K. and Sukhatme, G. (2002) Connecting the Physical World with Pervasive Networks. IEEE Pervasive Computing, 1, 59-69. http://dx.doi.org/10.1109/MPRV.2002.993145

[3] Akyildiz, F., Su, W., Sankarasubramaniam, Y. and Cayirci, E. (2002) A Survey on Sensor Networks. IEEE Communications Magazine, 40, 102-114. http://dx.doi.org/10.1109/MCOM.2002.1024422

[4] Heinzelman, W., Chandrakasan, A. and Balakrishnan, H. (2000) Energy-Efficient Communication Protocol for Wireless Sensor Networks. Proceedings of the 33rd Hawaii International Conference on System Sciences, 8, 8020. 
http://dx.doi.org/10.1109/HICSS.2000.926982

[5] Heinzelman, W., Chandrakasan, A. and Balakrishnan, H. (2002) An Application-Specific Protocol Architecture for Wireless Micro-Sensor Networks. IEEE Transactions on Wireless Communication, 1, 660-670. http://dx.doi.org/10.1109/TWC.2002.804190

[6] Lindsey, S. and Raghavendra, C.S. (2002) Power-Efficient Gathering in Sensor Information Systems. IEEE Aerospace Conference Proceeding, 3, 1125-1130. http://dx.doi.org/10.1109/aero.2002.1035242

[7] Pottie, D.J. and Kaiser, W.J. (2000) Wireless Integrated Network Sensors. Communication of the ACM, 43, 51-58. http://dx.doi.org/10.1145/332833.332838

[8] Li, W. and Chen, G. (2006) Energy-Efficient Clustering Algorithm in Wireless Sensor Network. IET International Conference, November 2006, 1-4. http://dx.doi.org/10.1016/s1005-8885(07)60015-6

[9] Salhiel, A., Weinmann, J., Kochhal, M. and Schwiebert, L. (2001) Power Efficient Topologies for Wireless Sensor Networks. International Conference on Parallel Processing, Valencia, 3-7 September 2001, 156-166.

[10] Wei, D. and Chan, A. (2006) Clustering Ad Hoc Networks: Schemes and Classifications. 2006 3rd Annual IEEE Communications Society on Sensor and Ad Hoc Communications and Networks, 3, 920-926. http://dx.doi.org/10.1109/SAHCN.2006.288583

[11] Al-Karaki, J.N. and Kamal, A.E. (2004) Routing Techniques in Wireless Sensor Network. IEEE Wireless Communication, 11, 6-28. http://dx.doi.org/10.1109/MWC.2004.1368893

[12] Abba Ari, A.A., Gueroui, A., Labraoui, N. and Yenke, B.O. (2015) Concepts and Evolution of Research in the Field of Wireless Sensor Networks. International Journal of Computer Networks \& Communications (IJCNC), 7, 81-98. http://dx.doi.org/10.5121/ijcnc.2015.7106

[13] Thangadurai, N. and Dhanasekaran, R. (2013) Energy Efficient Cluster Based Routing Protocol for Wireless Sensor Networks. International Journal of Computer Applications, 71, 43-48. http://dx.doi.org/10.5120/12374-8699

[14] Chissseenni, C.F., Monti, P. and Nucci, A. (2002) Energy Efficient Design of Wireless Ad Hoc Networks. Proceedings of European Wireless, 1.

[15] Rauthan, J.S. and Mishra, S. (2012) An Improved Cluster Based Multi-Hop Routing in Organizing Wireless Sensor Networks. International Journal of Engineering Research and Technology, 1, 1-9.

[16] Saravanakumar, R., Susila, S.G. and Raja, J. (2011) Energy Efficient Constant Cluster node scheduling Protocol for Wireless Sensor. WSEAS Transactions on Communications, 10, 119-128.

[17] Shwe, H.Y., Jiang, X.-H. and Horiguchi, S. (2009) Energy Saving in Wireless Sensor Networks. Journal of Communication and Computer, 6, 20-27.

[18] Shringi, S. and Taruna, S. (2013) A Cluster Based Routing Protocol for Prolonging Network Lifetime in Heterogeneous Wireless Sensor Networks. International Journal of Advanced Research in Computer Science and Software Engineering, 3, 658-665.

[19] Jebakumar, R. and Vivekanandhan, P. (2014) Distributed Data Storage through Secure Routing in WSNs. International Review on Computers and Software, 9, 478-486. 